\title{
Fashion and Health - Utilizing Wearable Technology and Traditional Chinese Medicine to Design Two Wearable Acupressure Prototypes
}

\author{
Chajuana Trawick ${ }^{1 *}$ and David Yeung ${ }^{2}$ \\ ${ }^{1}$ School of Arts, Media and Communications, Lindenwood University, USA \\ ${ }^{2}$ Winchester School of Art, University of Southampton, UK
}

*Corresponding author: Dr. Chajuana Trawick, Lindenwood University, 209 S.

Kingshighway, St. Charles, MO 63301, USA.

Received Date: August 01, 2019

Published Date: August 06, 2019

\begin{abstract}
According to Wright and Keith [1], "wearable technology and wearable devices are phrases that describe electronics and computers that are integrated into clothing and other accessories that can be worn comfortably on the body". Examples of wearable devices include; glasses, watches, headbands, and jewelry. These technologies have also been applied to fashion in relation to sport activities, fitness and even health. For instance, we can see heart rate monitors, personal GPS devices, smart watches or Bluetooth headsets in various markets. From the fashion approach, there are designers who have already incorporated similar forms of technology into cutting edge apparel. In spring/summer 2007, the Turkish born designer Hussein Chalayan, made robotic dresses which stunned the audience [2]. Mower mentioned that Chalayan was "excited by science, fascinated by world politics, and recognized the fact that the way we dress was a reaction to the times we lived in" [3]. In 2000, the brand 'Levis' worked with Philips to create a jacket which had special pockets for MP3 players, mobile phones and cables [4]. VHM, a design futures company, contended that this product "was widely considered to be the first commercial wearable electronics garment" [2]. In 2016, Nike worked with Apple to create "Apple Watch Nike+" for runners, which consisted of a functional watchband that was both breathable and sweat-resistant [3]. This article focuses on the combination of fashion and health by utilizing wearable technology and traditional Chinese medicine to design two wearable acupressure prototypes, based on the concept that "fashion can heal".
\end{abstract}

Keywords: Wearable technology; Fashion can heal; Traditional Chinese medicine; User-friendly, Health care

\section{Introduction}

Peltola wrote, that by approximately 2022 there will be over a billion wearable devices around the world, and many of these devices would be utilized by the healthcare industry to improve the quality of human life [5]. Such information was an inspiration to create fashion technology into this research. David Yeung, the lead researcher and creator of the prototypes, believes that fashion can be empathetic and help create functional purposes, instead of only being confined to the conventional concept of showing one's beauty or identity. While living in China, David found traditional Chinese medicine (TCM) to be interesting, with its long history and thousands of years of development. It was during this time in China, that the idea of "fashion can heal" emerged. In an article by Interesting Engineering, it was noted that the first wearable technology consisted of the 400-year-old abacus rings, used for trade in China and developed in the Qing Dynasty era, between
1644 and 1911 in China [6]. Since that time, computer technology has significantly increased.

Thorp, E. addressed that the first computer was conceived in 1955 [7]. In the summer of 1961, Thorp noted that the first wearable computer was operated for the purpose of a 'casino' test in Las Vegas, USA [7]. As time continues to pass, wearable technologies are being developed into different aims and functions, and the integration of health care and wearable technology is one of those developments [7]. Cicek noted, that there were three main categories of wearable technology: wearable health technologies, wearable textile technologies and wearable consumer electronics [8]. However, for this research report, the authors focused on, how wearable technologies can be associated with human health and traditional Chinese medicine to develop two wearable acupressure prototypes. The National Center for Complementary 
and Integrative Health mentioned that traditional Chinese medicine (TCM) originated in ancient China and has evolved over thousands of years [8]. They also noted that, TCM practitioners used herbal medicines and various mind and body practices, such as acupuncture and tai chi, to treat or prevent health problems [8]. According to Peltola, wearing healthcare devices were received more positively, by a higher number of people [5]. And, based on Collier and Randolph's, 2015 research, there had been a consistent combination of wearable technology within the fitness and medical industries [5]. The relationship between health and information technology has become more influential with technology becoming more pervasive in healthcare, ranging from individualized self-care and disease management, to improvements in hospital efficiency [5]. The aim of this project was to utilize wearable technology and traditional Chinese medicine to design two wearable acupressure prototypes to give human medical treatment for healing the body and spirit, founded on the idea of "fashion can heal".

\section{Discussion}

In this postmodern era, most consumers work under pressure to quickly adapt to the changing society and growing competitive work fields [9]. Overtime, consumers gradually forget about taking care of themselves and being healthy, either physically or mentally [9]. This type of unhealthy fast-paced energy is known as 'Qi' imbalance which weakens the immune system [9]. According to Reninger in ThoughtCo, 'Qi' is defined as a life-force, which animates the forms of the world [10]. Reninger also stressed that in Taoist cosmology, the two most fundamental forms of qi are Yinqi and Yang-qi, as they are the primordial feminine and masculine energies to balance life forms [10]. In relation to Yin-qi and Yang-qi, this research examined TCM healing methods and used acupressure as its guide, to develop two user friendly wearable acupressure prototypes. The concept of giving human medical treatment for healing the body and spirit, founded on the idea that "fashion can heal" was the motivation behind incorporating electronic systems into apparel materials (Figure 1). Chen, YW and Wang HH remarked that acupressure was a complementary treatment that used fingers and hands to stimulate acupoints and maintain the balance of energy [9]. By using the two wearable acupressure prototypes, it was expected that the system could regularly help release stress and raise energy levels to fight against illness [11]. Additionally, users could use an electronic system to perform massages rather than by hand or fingers [11].

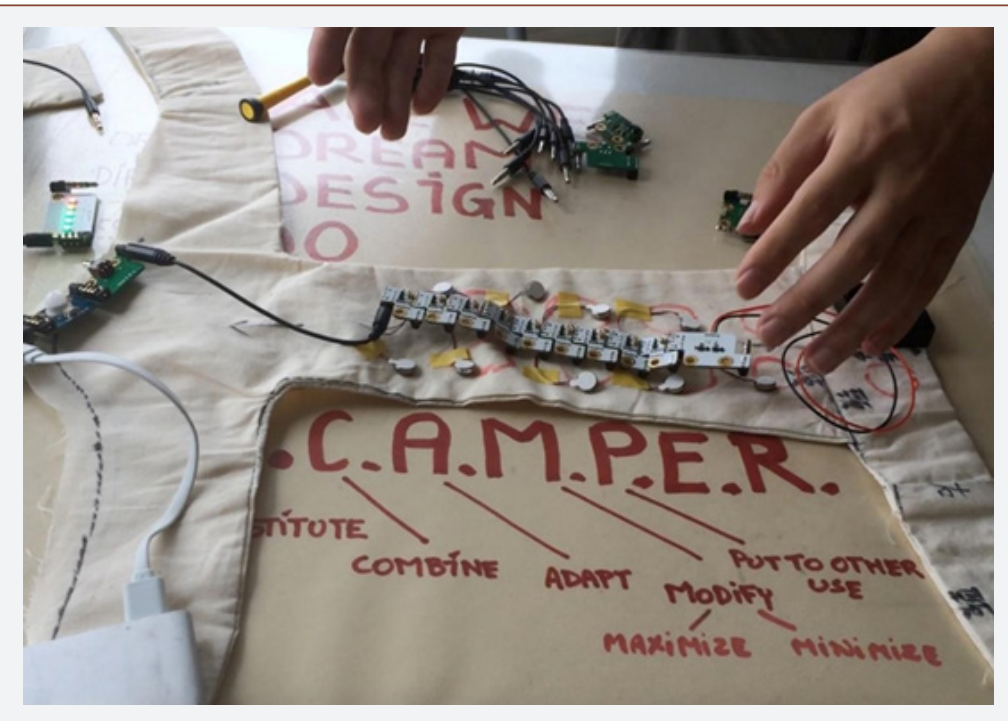

Figure 1: The use of the electronic system 'Jack Brick' in connection with fabric materials.
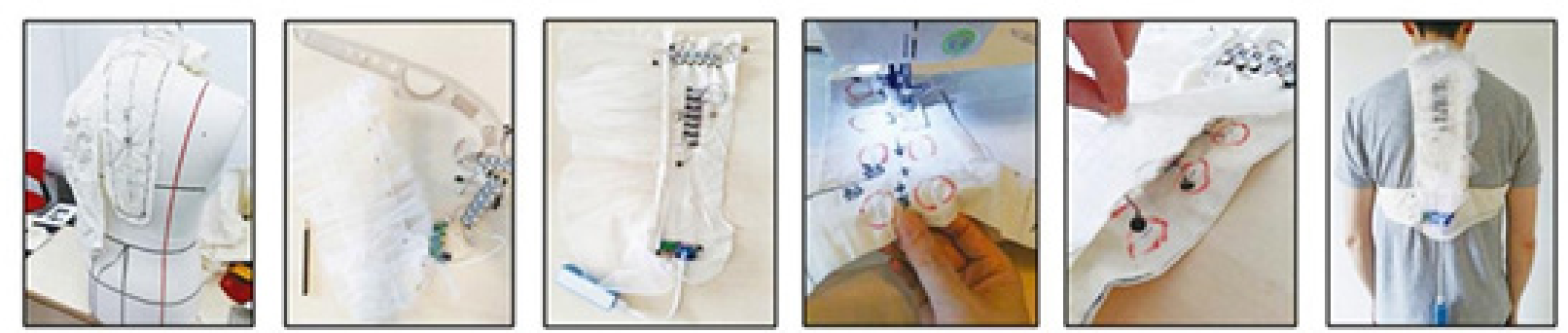

Figure 2: The process and development of the wearable apparel technology prototype.

With resources and support provided by Jack Brick Systems, a technology company, who developed an electronic system named 'Jack Brick', David was able to utilize the same 'Jack Brick' system to develop two wearable acupressure prototypes [12] (Figure 2).
Bricks with vibrating functions that could be placed on top of the acupoints within the prototypes, were chosen. The system was then embedded into the surface of fabric materials, so users could simply regulate the on and off switch using a remote-control device, smart 
phone or related system controller. When turned on, the bricks would create vibrations along the acupoints, to create acupressure while wearing either of the two wearable prototypes (Figure 2).

Once the vibrating system was embedded, the next step was to add the illuminated bricks. The illumination system, also in brick shape, were part of the Jack Brick invention as well [12]. When connected to the vibrating bricks, the illumination effect would change into different colors. Different colored lights within the illumination were chosen to indicate which area was under the massage treatment. The illuminating bricks would also allow users to know what level of vibration would be achieved and which part of the area would be treated when using the devices (Figure 3). The illumination system could also be turned on and off by remote control or smartphone with a downloaded app.

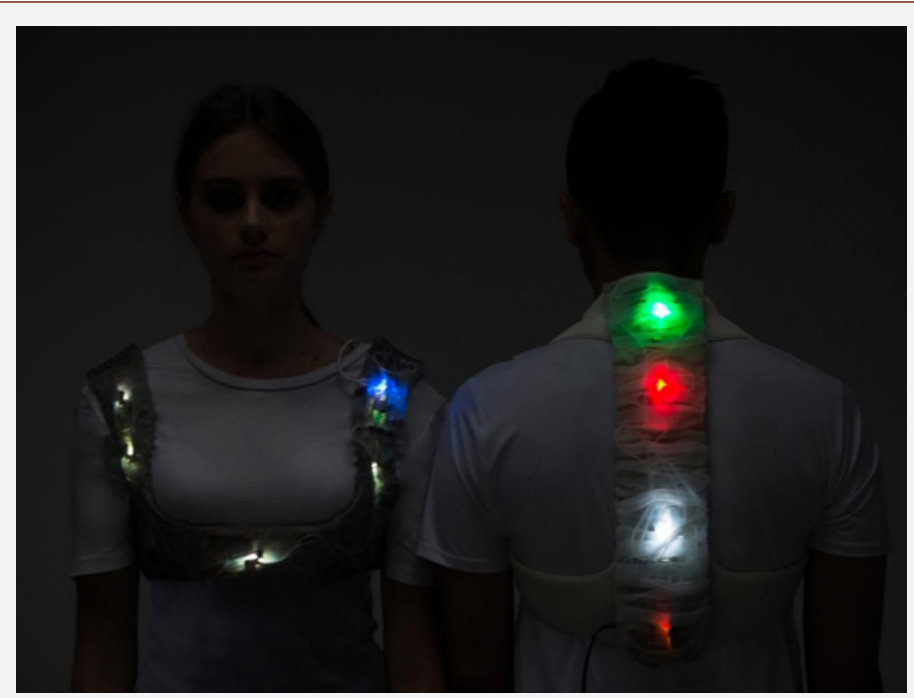

Figure 3: Illumination effect is seen when they are in darkness.

Utilizing the Jack Brick system, two wearable acupressure prototypes were designed to target two areas identified as important when using TCM methods to relive stress and improve health; the backbone and under the breast area. In the first design, the vibrating and illuminating system was placed along the backbone (Figure 1). Focusing on the backbone was key, due to the number of acupressure points connected to different parts of the organs in the body. The vibrating and messaging devices would strengthen the immune system and allow the body to maintain a consistent balance of energy [9]. With a successful outcome, healing the physical and mental state of the user, would be achieved through the use of TCM methods and the wearable acupressure prototypes, founded on the idea that "Fashion can heal".

In the second design, the messaging system was placed under the breast area (Figures 4\&5). The regular application of the massaging device was intended for daily use on target areas to raise positive energy in the immune system as related to Yin-qi and Yangqi [10]. The area under the breast area was targeted, due to the large number of lymphatic glands which help get rid of toxins, waste and unwanted materials. It is believed that regularly massaging these areas of the body could reduce toxins and stress and increase good health [10].

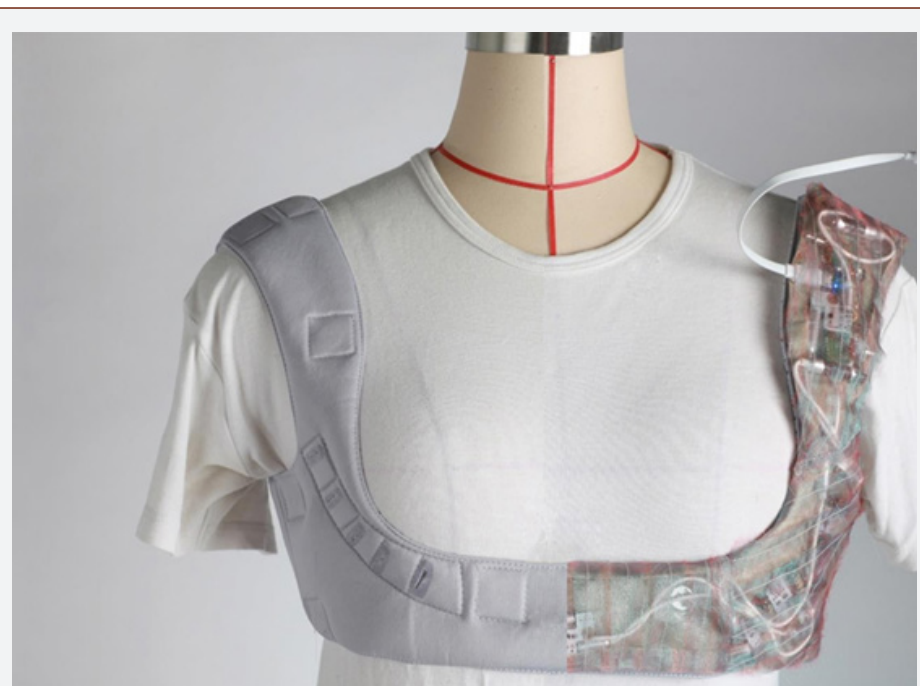

Figure 4: The finished second prototype designed to go under the breast area. The inside of the device is exposed on the right and covered on the left. 
Figure 5: The first finished prototype designed for the 'backbone'.

\section{Conclusions}

Features of the wearable acupressure prototypes devices can be summarized as follows:

1. Make use of traditional Chinese medical treatment to heal

2. User friendly

3. Portable and detachable

4. Technologically advanced,

5. Physical and/or mental relief

Users could physically carry the device from one place to another and put it on to achieve the healing function or medical treatment at any time. The device would be user-friendly and could be put on without assistance. To activate the system a power bank and cable would be needed, similar to charging a cellular phone. In addition, the system devices would be detachable in order to wash the fabric materials for hygienic purposes. The main fabric components of the device were cotton, neoprene and organza. The cotton and neoprene materials worked as a base and were sturdy in structure to support the entire body of the prototypes. The brick system was on top of the device and was embedded into the cotton fabric. Due to the lightweight and semi see-through characteristics of organza, it was used for two purposes. The organza covered the brick system and allowed the system to sense signals coming through the device. The organza also allowed illumination of the signals to appear when the system was on. Additionally, the organza could be sterilized by spraying alcohol onto the fabric for cleaning purposes. To conclude, the wearable acupressure prototypes are functional, ergonomic, aesthetically designed, and technologically detailed. However, limitations due to resources and financial support decreased production aspects and testing of the prototypes. Specifically, the cotton and neoprene, although sturdy, were not as durable as expected.

Using the fabric materials alongside the electronic brick system was also challenging, because of their extreme differences in weight, hand, and properties. Further research would be needed to determine the best fabric to use based on comfort, function, and visibility. The brick system would also need to be enhanced to increase the effectiveness and strength of the vibration signals through the fabric materials. Although there were a few limitations, the overall creation of the wearable acupressure prototypes was satisfactory. With full sponsorship provided for the brick devices by the Jack Brick Systems company, the wearable acupressure prototypes were realized. When considering the concept "Fashion can heal" apparel design educators and practitioners could develop similar devices that would incorporate both fashion and health for the growth and benefit of society.

\section{Acknowledgement}

None.

\section{Conflict of Interest}

Authors declare no conflict of interest.

\section{References}

1. Wright R, Keith L (2014) Wearable Technology: If the tech fits, wear it. Journal of Electronic Resources in Medical Libraries 11(4): 204-216. 
2. Peltola $O$ (2017) Introduction to wearable healthcare technology.

3. Mower S (2006) Spring 2007 ready-to-wear Chalayan.

4. VHM (2019) 2000 Philips/Levis ICD+.

5. Apple (2019) Apple Watch Nike+.

6. Interesting engineering (2019) The smart ring: from the 17th century wearable abacus to today.

7. Thorp E (1999) The invention of the first wearable computer, Digest of Papers, Second International Symposium on wearable computers, Cat. No.98EX215.
8. National Center for Complementary and Integrative Health (2019) 'Traditional Chinese Medicine: In depth'.

9. Chen YW, Wang HH (2012) The effectiveness of acupressure on relieving pain: a systematic review. Pain Manag Nurs 15(2): 539-550.

10. Reninger E (2017) Qi (Chi): The Taoist principle of life force.

11. Research Gate (2019) Wearable technologies and its future applications.

12. Jack Brick Systems (2017). 